\title{
Sound prescriptions to mitigate the effects of COVID-19 in the population with Down syndrome
}

\author{
Sergio Verd ${ }^{1,2}$ (I) $\cdot$ Isabel Vinuela ${ }^{3} \cdot$ Mateo Verd $^{4}$ \\ Received: 6 March 2021 / Accepted: 8 May 2021 / Published online: 26 May 2021 \\ (C) Fondazione Società Italiana di Neurologia 2021
}

To the Editor,

Altable and de la Serna [1] have reviewed a wide spectrum of mechanisms leading to the immune dysregulation that affects persons with Down syndrome (DS). These anomalies potentially explain why children with DS present a higher risk of respiratory infections, while adults with DS experience an increased immune response characterised by a high oxidative stress. Accordingly, Altable and de la Serna anticipate a more severe COVID-19 progression in patients with DS compared with controls. At the time of writing their report, cases of COVID19 in patients with DS had been described, but no robust clinical or epidemiological data were available, regarding COVID19 characteristics in people with DS. Therefore, the Altable and de la Serna's report prepared early in the epidemic appears to have been remarkably accurate in its prediction of which patients would be prone to life-threatening COVID-19.

We know now that DS over age 40 boosts the mortality risk of hospitalised patients with COVID-19 to $51 \%$; and a recent study on primary care data from above 8 million adults in the UK has reported that if infected, people with DS are ten times more likely to die than the general population [2]. This data is especially striking considering that very severely frail individuals with COVID-19 are only three times more likely to die than those who are not frail [3]. These facts give a measure of the disproportionate impact of COVID-19 on DS persons.

Sergio Verd

drsverd@gmail.com

1 Pediatric Unit, Department of Primary Care, La Vileta Surgery, Matamusinos Street, 07013 Palma de Mallorca, Spain

2 Balearic Islands Health Research Institute (IdISBa), 79 Valldemossa Road, 07120 Palma de Mallorca, Spain

3 Department of Paediatrics, Son Espases University Hospital, 79 Valldemossa Road, 07120 Palma de Mallorca, Spain

4 Department of Anesthesiology, Perioperative and Pain Medicine, Son Espases University Hospital, 79 Valldemossa Road, 07120 Palma de Mallorca, Spain
Given that it has been ascertained that people with DS stand out among groups at higher risk of dying from COVID-19, there must be no further delay in putting into practice specialised care for them. At this point, it seems apposite to express some words of caution with regard to the antipyretic medication of these patients. This proposal falls within a more ambitious frame aiming at critically reviewing all medications linked to increased rates of respiratory infection or hospitalisation during the present COVID-19 pandemic. A trial in support of this view is the large case-control study of all 4251 cases of severe COVID-19 in Scotland since the start of the epidemic [4], which unveils that prior prescribing of non-opioid analgesics was very significantly associated with elevated rate ratios for severe COVID-19 $\left(\mathrm{P}=10^{-12}\right)$.

The oxidative stress that leads to deadly inflammation in severe COVID-19 may be counterbalanced by reduced glutathione (GSH), a key antioxidant molecule ubiquitous in all tissues. Paracetamol is the best-known drug to lower GSH levels; more precisely, plasma GSH becomes significantly reduced after a single $2 \mathrm{~g}$ paracetamol administration. As the pool of the available GSH molecules is fixed, any increase of its utilisation can leave patients with chronic low-grade inflammation unprotected from a disease that can prove fatal. As part of this approach, a very recent research has reported that not taking paracetamol versus taking paracetamol cuts the relative risk of respiratory support of COVID-19 patients by $84 \%$ [5].

At a dose of $20 \mathrm{mg} / \mathrm{kg}$ of paracetamol, DS patients exhibit a characteristic shift toward glutathione-derived metabolites, and GSH depletion, which can leave the organism defenceless to fight the raging inflammation of COVID-19. Taken together, current practice that considers the liberal use of paracetamol a necessity, and the lifelong chronic immune hyperactivity that is part of the fundamental biology of DS, may confer a very high risk of death from COVID-19 to these patients.

We would like to emphasise that paracetamol was included in the list of widely prescribed drugs of concern as "medications compromising COVID", since these are often 
ineffective, given for unnecessarily long periods, at wrong doses, and it is getting obvious that where possible should be deprescribed [4]. To conclude, it is now time to call against the indiscriminate use of paracetamol to treat patients with COVID-19 and Down syndrome. Under the present circumstances, these must be things we do for good reasons.

\section{Declarations}

Conflict of interest The authors declare no competing interests.

Ethical approval None

\section{References}

1. Altable M, de la Serna JM (2021) Down's syndrome and COVID19: risk or protection factor against infection? A molecular and genetic approach. Neurol Sci 42:407-413. https://doi.org/10.1007/ s10072-020-04880-x

2. Clift AK, Coupland CAC, Keogh RH, Hemingway H, HippisleyCox J (2020) COVID-19 mortality risk in Down syndrome: results from a cohort study of 8 million adults. Ann Intern Med M20:4986. https://doi.org/10.7326/M20-4986

3. Geriatric Medicine Research Collaborative (2021) Age and frailty are independently associated with increased COVID-19 mortality and increased care needs in survivors: results of an international multi-centre study. Age Ageing: afab026. doi: 10.1093/ageing/ afab026.

4. McKeigue PM, Kennedy S, Weir A et al (2021) Relation of severe COVID-19 to polypharmacy and prescribing of psychotropic drugs: the REACT-SCOT case-control study. BMC Med 19:51. https://doi. org/10.1186/s12916-021-01907-8

5. Rinott E, Kozer E, Shapira Y, Bar-Haim A, Youngster I (2020). Ibuprofen use and clinical outcomes in COVID-19 patients. Clin Microbiol Infect 26:1259.e5-1259.e7. doi: https://doi.org/10.1016/j. cmi.2020.06.003.

Publisher's note Springer Nature remains neutral with regard to jurisdictional claims in published maps and institutional affiliations. 